\title{
Actitud hacia la Inclusión de los Estudiantes de Pedagogía de una Universidad Estatal Chilena
}

\author{
Attitude to the Inclusion of Pedagogy Students of a Chilean \\ State University
}

\author{
Pablo Castillo Armijo * \\ Carlos Miranda Carvajal
}

Facultad de Ciencias de la Educación, Universidad de Talca, Chile

\begin{abstract}
Las actuales políticas públicas en materias de educación del Estado chileno han declarado dentro sus ejes claves la inclusión educativa y una nueva educación pública de calidad que brinde las mejores oportunidades a todos sus habitantes, en especial a los más vulnerados social, cultural y económicamente. De esta manera se asume en plenitud el compromiso internacional mandatado por la Organización de Naciones Unidas en la Agenda 2030 para el Desarrollo Sostenible, en particular el Objetivo de Desarrollo Sostenible 4, que busca garantizar una educación inclusiva y equitativa de calidad y promover oportunidades de aprendizaje permanente para todos (ONU/CEPAL, 2016). La presente investigación plantea el objetivo de indagar sobre las actitudes de estudiantes de pedagogía para promover el desarrollo de escuelas inclusivas. Es necesario, por tanto, conocer lo que piensan y sienten uno de sus principales actores que liderarán estos cambios, como son los futuros profesores. El estudio se desarrolló bajo un modelo cuantitativo, multivariado, descriptivo y correlacional del fenómeno a partir de las percepciones generales de la muestra según el constructo estudiado. La recolección de datos se realizó por medio de la adaptación a la realidad chilena del "Cuestionario para futuros docentes de Educación Secundaria acerca de las percepciones sobre atención a la diversidad” (Colmenero y Pegalajar, 2015). Los resultados arrojan una percepción positiva hacia la inclusión por parte de los estudiantes, pero falta materializarla en una mejor formación inicial docente y en prácticas reales de inclusión.
\end{abstract}

Descriptores: Educación inclusiva; Formación de docentes; Análisis cuantitativo.

The current public policies in education matters of the Chilean State have declared within their focus the educational inclusion and a new public quality education that offers the best opportunities to all its inhabitants, especially to the most vulnerable socially, culturally and economically. In this way, takes the international commitment mandated by the United Nations in the 2030 Agenda for Sustainable Development, in particular the Sustainable Development Goal 4, whose objective is to guarantee an inclusive and equitable quality education and to promote opportunities of permanent learning for all (UN/CEPAL, 2016). The objective of this investigation considers studying the attitudes of pedagogy students to promote the inclusive school's development. It is necessary, therefore, to know what they think and feel one of their main actors that will lead these changes, future teachers. This study was developed under a quantitative, multivariate, descriptive and correlational model of the phenomenon based on the general perceptions of the sample according to the studied construct. The data collection was carried out through the adaptation to the Chilean reality of the "Cuestionario para futuros docentes de Educación Secundaria acerca de las percepciones sobre atención a la diversidad" (Colmenero \& Pegalajar, 2015). The results show a positive perception towards inclusion of students, but it is necessary materialized them in a better initial teacher training and in real inclusion practices.

Keywords: Inclusive education; Teacher educator training; Quantitative analysis.

*Contacto: pablo.castillo.armijo@gmail.com

ISSN: 0718-7378

www.rinace.net/rlei/
Recibido: $\quad 10 / 08 / 2018$

$1^{a}$ Evaluación: 24/08/2018

Aceptado: $\quad 04 / 10 / 2018$ 


\section{Introducción}

La atención de los estudiantes que presentan necesidades educativas especiales (NEE) por muchos años ha estado a cargo de especialistas como fonoaudiólogos, terapeutas ocupacionales, profesores diferenciales entre otros (MINEDUC, 2007), esta tendencia se ha mantenido los últimos años y muchos de los fondos de la ley de Subvención Escolar Preferencial se han orientado hacia el Programa de Integración Escolar que mantiene la idea de apoyos pedagógicos en estos profesionales que colaboran con los profesores dentro del aula en el tratamiento de estos estudiantes clínicamente diagnosticados y donde se establece una hibridez de las políticas de inclusión social y en especial en el actual sistema educativo donde conviven en paralelo la idea de integración con el nuevo paradigma de la inclusión (López et al., 2012).

En la investigación de Tenorio (2011) realizada a futuros profesores de enseñanza básica reconocen como una debilidad la falta de preparación en temas relacionados con diversidad en el aula, integración escolar y estrategias pedagógicas para estudiantes con NEE. Así mismo, Chiner (2011), luego de una revisión sobre investigaciones de percepciones y actitudes del profesorado hacia la inclusión de alumnado con NEE en el mundo anglosajón e hispano, logra establecer una aceptación general a la idea de inclusión, aunque el apoyo disminuye a la hora de implicarse y favorecer prácticas reales de inclusión en las aulas. Entre las razones entregadas en esa disminución se mencionan la falta de tiempo, la falta de preparación o formación y los recursos para atender a estudiantes tan diversos. La falta de preparación también es destacada por la investigación de González-Gil y colaboradores (2016) cuando manifiestan la necesidad de mejorar los programas de formación docente para que aprendan a trabajar con todo el alumnado y en contextos de amplia diversidad.

Para el caso chileno la investigación de Sanhueza, Granada y Bravo (2012), establecen que los profesores participantes del estudio apoyan decididamente la idea que la educación inclusiva posee beneficios sociales, sin embargo, la percepción disminuye cuando se plantea un beneficio académico para todo el alumnado. Los criterios de desempeño profesional de los profesores chilenos están descritos y determinados en el Marco para la Buena Enseñanza (MINEDUC, 2008) con un énfasis en el logro de los aprendizajes de los estudiantes. Los profesores asumen diversas responsabilidades pedagógicas dentro de este marco, como identificar fortalezas y debilidades en el aprendizaje de los estudiantes, crear climas de convivencia y desafíos de aprendizajes significativos y también involucrar a las familias dentro de la comunidad educativa, pero no se hace alusión explícita a la creación de estrategias para el trabajo hacia la inclusión educativa lo que plantea un desafío para la formación inicial y permanente de nuestros profesionales de la educación.

A partir del año 2015 se aprobó la Ley de Inclusión (MINEDUC, 2015) que regula la admisión de los y las estudiantes, elimina el financiamiento compartido y prohíbe el lucro en establecimientos educacionales que reciben aportes del Estado comenzando un proceso nuevo para la gestión de los centros educativos en materia de integración, inclusión y atención a la diversidad. Los retos de incorporar prácticas inclusivas en el centro educativo deben ser asumidos por todos sus actores y se requerirá información y formación para lograr los objetivos que persigue la ley (González-Gil et al., 2016). Esta tensión debe ser un motivo de investigación e intervención para las políticas públicas.

La OCDE (2009) reconoce a los profesores como el principal factor para lograr mejores aprendizajes en sus estudiantes, de allí radica la necesidad de enfocarnos en sus actitudes 
hacia la inclusión. Entenderemos, por tanto, por actitud como aquella predisposición que afecta consistentemente la respuesta de las personas hacia determinados objetos o situaciones. Las actitudes definen los procesos de pensamiento, acción y predisposición al cambio ante determinados retos (Richardson, 1996). Los retos de inclusión superan los límites de la escuela y colaboran a mejorar nuestra sociedad. Es así que los actuales fenómenos de migración, interculturalidad, atención a estudiantes con necesidades educativas especiales entre otros son desafíos para los centros de educación superior que forman profesores.

\section{Revisión de la literatura}

\subsection{Movimiento histórico por una educación para todos}

El primer hito para avanzar en la inclusión educativa que se declaran hoy como necesidad sentida en muchos países en vías de desarrollo, entre ellos Chile, tuvo su origen en la Declaración Mundial sobre Educación Para Todos desarrollada en Jomtien (Tailandia), donde se reunieron 155 Estados en 1990 para levantar la voz de los que por muchos años permanecieron al margen de la sociedad, sean estos niños y niñas y personas jóvenes y adultas privadas de una educación básica por distintas condiciones: inmigrantes, trabajadores, poblaciones de zonas remotas y rurales, desplazados por las guerras, refugiados, minorías étnicas, raciales y lingüísticas y pueblos sometidos a un régimen de ocupación (UNESCO, 1990). Esta declaración estableció como prioridad universalizar el acceso a la educación básica, fomentar la equidad, prestar atención prioritaria y construir un ambiente favorecedor al aprendizaje y respetar el derecho esencial que tienen todas las personas a la educación.

Se reconoce en ella que "la educación puede contribuir a lograr un mundo más seguro, más sano, más próspero y ambientalmente más puro y que al mismo tiempo favorece el progreso social, económico y cultural, la tolerancia y la cooperación internacional" (UNESCO, 1990, p. 6), pero también que no será fácil conseguir estos objetivos sin un compromiso a largo plazo por todas las sociedades, en especial por las más desarrolladas para contribuir de manera activa en la lucha contra la pobreza y desigualdad social. Es así, que durante los años siguientes se sucederán variadas conferencias para reafirmar esta primera declaración de una Educación Para Todos (EPT), recordándoles a los Estados firmantes y organizaciones colaboradores los compromisos asumidos.

Con la declaración sobre Necesidades Educativas Especiales: Acceso y Calidad de Salamanca (UNESCO, 1994) se dio un salto cualitativo al proceso político y educativo de una EPT, al considerar la integración de vastos sectores de la población escolar que por años fueron postergados y marginados, como fueron las personas con NEE. En esta declaración de Salamanca se reconoce que todos los niños, de ambos sexos, tienen el derecho fundamental a la educación en escuelas ordinarias y que éstas deben diseñar programas que reconozcan las características, intereses, capacidades y necesidades de aprendizaje que les son propias a cada individuo. También se incorpora como idea fuerza la participación y colaboración de las familias al interior de los centros educativos y la de garantizar programas de formación inicial y permanente del profesorado acorde con las nuevas demandas de una escuela integradora.

Dentro del Marco de Acción establecido para llevar a la práctica esta declaración se demanda que, 
las escuelas deben acoger a todos los niños, independientemente de sus condiciones fïsicas, intelectuales, sociales, emocionales, lingüísticas u otras. Deben acoger a niños discapacitados y niños bien dotados a niños que viven en la calle y que trabajan niños de poblaciones remotas o nómadas, niños de minorías lingüísticas étnicas o culturales y niños de otros grupos o zonas desfavorecidos o marginados. (UNESCO, 1994, p. 6)

El sueño de una educación para todos comenzó a cobrar mayor fuerza al incluir a muchas más personas que, de una u otra manera, han sido excluidas sistemáticamente dentro de sus sociedades. Los desafíos de inclusión han quedado planteados desde aquel momento para los encargados de diseñar las políticas educativas y principalmente para el sistema educativo y los centros escolares que deberán modificar sus prácticas en favor de la atención a la nueva diversidad y a las exigencias educativas producto de la masificación de la educación en las últimas tres décadas (Esteve, 2003). El Foro Mundial sobre la Educación para Todos: cumplir nuestros compromisos comunes, celebrado en Dakar (UNESCO, 2000) reafirmó esa idea de acceso igualitario y atención preferencial a aquellos estudiantes con mayores necesidades y más vulnerables. Se estableció como acuerdo un plazo de 15 años para consolidar y lograr el objetivo de una educación básica para todos y la igualdad entre los géneros en el ámbito educativo.

La Conferencia Internacional de Educación 48 ${ }^{a}$ Reunión titulada La Educación Inclusiva: Un camino hacia el futuro celebrada en Ginebra en 2008 a mitad del proceso para conseguir la EPT los Estados miembros y organizaciones intergubernamentales, las ONG's e instituciones de la sociedad civil afirman que una educación inclusiva, además debe ser de calidad, equitativa y efectiva, actualizando de esta manera el concepto de una educación de calidad para todos (UNESCO, 2009). Se supera la idea que sólo con el acceso y la cobertura se lograrían las metas de alcanzar el desarrollo humano, social y económico y se avanza hacia lo que actualmente entendemos por el concepto de inclusión educativa que es bastante mayor que la idea de asimilación, integración o tolerancia que se planteaba en el comienzo de la década de los noventa.

Las principales recomendaciones entregadas a los Estados miembros, fruto de las 4 discusiones regionales desarrolladas previamente a la conferencia, abarcaron varias áreas dando cuenta del alcance que se persigue esta política de inclusión liderada por la UNESCO. En cuanto al enfoque y alcance del concepto se reconoce que,

\section{la educación inclusiva es un proceso permanente, cuyo objetivo es ofrecer una educación de calidad para todos, respetando la diversidad y las distintas necesidades y aptitudes, características y expectativas de aprendizaje de los educandos y de las comunidades, eliminando toda forma de discriminación. (UNESCO, 2009, p. 19)}

Además, se menciona la lucha contra la pobreza y desigualdad social y la necesidad de promover entornos escolares y culturales que respeten la igualdad de género y la participación de los propios educandos, sus familias y sus comunidades. Para las políticas públicas hace un llamado a recabar información sobre las diversas formas de exclusión que afectan a las personas, en especial en el contexto escolar, donde se deben diversificar las prácticas educativas en calidad y equidad. Se deben, por tanto, diseñar marcos curriculares efectivos desde la infancia en adelante y formular políticas de apoyo pedagógico tendientes a reformas educativas encaminadas a la inclusión y que desarrollen mecanismos nacionales de seguimiento y aseguramiento de la calidad. Destaca el papel de liderazgo que deben tener los gobiernos en la promoción de la inclusión, velando por la participación y consulta a todas las partes interesadas para generar un compromiso social amplio que refuerce, por ejemplo, los vínculos entre las escuelas y las familias y que éstas puedan contribuir al proceso educativo de sus hijos. 
También existen recomendaciones para la mejora del estatus y condiciones de trabajo de los docentes, ya que a ellos les toca un rol fundamental para sensibilizar y educar en y para la inclusión. El cambio de paradigma sólo se logrará con el compromiso de todo el sistema educativo, y esto incluye a la formación continua de los docentes sobre prácticas y aprendizajes hacia una educación inclusiva (Essomba, 2006). Se deberá por tanto promover la investigación sobre la misma e instancias de trabajo con los demás actores educativos del acto educativo, establece el mandato de la conferencia.

Finamente destaca en este recorrido del movimiento para una Educación Para Todos (EPT) la declaración de la ONU el año 2015, con acuerdo de 193 países para avanzar en la Agenda 2030 para el Desarrollo Sostenible.

El objetivo 4 de dicha agenda busca garantizar una educación inclusiva, equitativa y de calidad y promover oportunidades de aprendizaje durante toda la vida para todos a partir del desarrollo de las metas como: educación primaria y secundaria universal, acceso a servicios de atención y desarrollo en la primera infancia y educación preescolar, acceso igualitario a la educación técnico-profesional y superior de calidad, aumentar las competencias necesarias para acceder a un trabajo decente, eliminar las disparidades de género en la educación y asegurar el acceso igualitario a todos los niveles de la enseñanza y la formación profesional para las personas vulnerables, incluidas las personas con discapacidad, los pueblos indígenas y los niños en situaciones de vulnerabilidad, alfabetización y jóvenes y adultos y una mejor educación cívica que defienda los derechos humanos y cree una cultura de educación para la paz y valoración de la diversidad cultural (ONU/CEPAL, 2016).

El recorrido histórico de estas múltiples conferencias y acuerdos internacionales destacan la importancia de valorizar la educación como un medio para el desarrollo sostenible del mundo, pero también nos hace tomar conciencia de la necesidad de transformación de nuestras prácticas pedagógicas en un mundo cada vez más complejo y cambiante. Debemos replantearnos lo que entendemos por educación (UNESCO, 2015) y por ende de lo que representa la escuela y sus principales actores.

\subsection{Importancia de una formación docente desde, hacia y para la inclusión educativa}

Desde la Conferencia Internacional de Educación $48^{\text {a }}$ Reunión sobre educación inclusiva, podemos apreciar con mayor fuerza la necesidad formativa que requieren los profesores para concretar el sueño de una educación inclusiva,

que formen a los docentes dotándoles de las capacidades y los materiales necesarios
para enseñar a distintas poblaciones estudiantiles y satisfacer las distintas necesidades
de aprendizaje de las diferentes categorías de educandos, mediante métodos como el
desarrollo profesional a nivel de la escuela, la formación inicial sobre inclusión y una
instrucción en la que se tenga en cuenta el desarrollo y los puntos fuertes de cada
educando. (UNESCO, 2009, p. 21 )

Los centros de educación superior formadores de profesores se han visto afectados por la inclusión educativa como idea que busca transformar las prácticas pedagógicas y han recibido demandas en relación con incorporar nuevos conceptos como NEE, diversidad, interculturalidad, integración e inclusión, además de cambios a nivel de mallas curriculares y programas de formación en educación básica, parvularia y carreras de especialidad (Infante, 2010). 
La Formación Inicial Docente es responsable de revisar y armonizar los planes de formación de las carreras de pedagogía para dar coherencia e integración entre trayectoria de aprendizaje, módulos, syllabus, entre otros. En favor de mejorar la calidad como también el desarrollo de capacidades tendientes a la inclusión, tanto en sus aspectos teóricos, pero también prácticos como parte del quehacer cotidiano de las prácticas pedagógicas. Los profesores y profesoras del sistema escolar son responsables, por su parte, de la ejecución de las actuales políticas públicas en materia de inclusión (Ley 20.845), y también de crear las condiciones para una escuela sin barreras a partir de la actualización de sus proyectos internos (educativo, convivencia, evaluación, inclusión entre otros) y para lograr aquellos desafíos requieren de una formación permanente que los capacite en el óptimo ejercicio de su profesionalidad.

Los autores más representativos de la inclusión educativa coinciden en señalar la formación de profesores como una piedra angular en las escuelas inclusivas. Nada se hará si no contamos con un profesorado bien formado en la dinámica de inclusión educativa (Escribano y Martínez, 2013). La investigación realizada por González-Gil y colaboradores (2016) concluye que las actitudes hacia la inclusión del profesorado son muy positivas y con altas expectativas en todos sus alumnos, pero no saben o muestran resistencia a la hora de modificar sus prácticas educativas cotidianas en favor de la inclusión, ya sea por falta de tiempo, escasez de recursos y de apoyos de la administración educativa y familias además de trabas organizativas de la propia institución escolar. En este mismo sentido, reconocen que no se encuentran lo suficientemente preparados, a nivel de formación, para hacerse cargo del desafío de la inclusión y atención a la diversidad de estudiantes en general.

En el caso chileno los especialistas formados en temas de inclusión son los educadores especiales o diferenciales, pero los actuales desafíos para todas las instituciones formadoras de profesores es hacerse cargo la toda la complejidad del sistema educativo para garantizar el derecho a la educación de todos y todas (Manghi et al., 2012).

\subsection{Concepto de inclusión educativa}

El concepto de inclusión fue representado en un comienzo al de asimilación y luego de integración, debido a que sus orígenes están ligados a la educación especial tradicional. La noción limitada que aporta esta mirada de la realidad sustentada en una visión médica de la diferencia orientó las políticas educativas de los Estados durante las décadas de los ochenta y noventa, principalmente en Europa y América del Norte, según Slee (2001).

El concepto de inclusión para Echeita y Sandoval (2002) está referido al derecho que tienen todos los niños y personas, no sólo de aquellos con necesidades educativas especiales a beneficiarse de la educación para no quedar excluidos de la escuela ni la cultura y sociedad en general. Frenar la exclusión social nos ayuda a tener por tanto mayor dignidad e igualdad, derechos humanos fundamentales. Misma opinión reflejan Stainback y Stainback (1999) cuando establecen que se ha producido un cambio en las escuelas corrientes que antes centraban sus esfuerzos en integrar y suplir las necesidades de los estudiantes con discapacidad, pero ahora el centro se ha ampliado a la atención de todos los miembros de la comunidad educativa aumentando de esta forma las posibilidades de mayor cohesión social. 
Cabe recordar que a fines de la década del noventa se ha superado el concepto de integración de estudiantes con NEE y se ha avanzado a paso firme hacia la inclusión educativa,

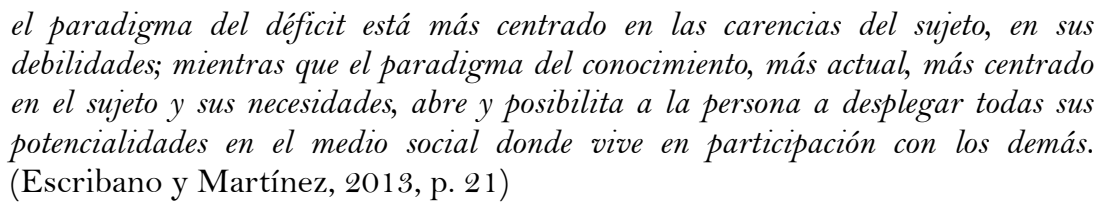

el paradigma del déficit está más centrado en las carencias del sujeto, en sus debilidades; mientras que el paradigma del conocimiento, más actual, más centrado en el sujeto y sus necesidades, abre y posibilita a la persona a desplegar todas sus potencialidades en el medio social donde vive en participación con los demás. (Escribano y Martínez, 2013, p. 21)

Ainscow, Booth y Dyson (2006) definen a la inclusión educativa como un proceso de mejora continuo que deben enfrentar las instituciones para diagnosticar sus barreras exclusoras y así poder eliminarlas y favorecer el aprendizaje y la participación de los estudiantes. En este mismo sentido es que la UNESCO (2005) definirá a la inclusión educativa como un proceso que intenta responder a la diversidad de necesidades de los estudiantes a partir de prácticas en las escuelas, la cultura y las comunidades reduciendo de esta forma la exclusión.

El concepto mismo de inclusión educativa hoy dependerá por tanto de lo que sus actores, al interior de las comunidades, los signifiquen tanto en sus discursos como en sus prácticas, de ahí surge la necesidad de conocer por dentro lo que piensan, lo que creen y lo que hacen todos los actores al interior de las aulas y centros educativos, develar los modelos de inclusión localmente situados (Mateus et al., 2017). Las barreras de exclusión y procesos de participación de los estudiantes en el caso chileno es un desafío y las actuales investigaciones deben enfocarse en sus actores. La educación inclusiva para las políticas públicas debe ser una prioridad ya que con ellas no solo transformamos y mejoramos la escuela, sino que a la propia sociedad (Slee y Allan, 2001).

Los objetivos de la investigación son:

- Identificar la actitud hacia la inclusión educativa de los estudiantes de pedagogía de una universidad pública chilena.

- Identificar el peso cuantitativo de las variables: elementos condicionantes del proceso de inclusión educativa, evaluación de la formación docente en relación a la inclusión educativa y práctica docente formativa hacia la inclusión educativa dentro de la actitud hacia la inclusión educativa de estudiantes en formación inicial docente de una universidad pública chilena.

- Identificar los niveles actitudinales hacia la inclusión educativa y diferencias existentes por: carreras y años de ingreso a la educación superior en una universidad pública chilena.

\section{Método}

El estudio se desarrolló bajo un modelo cuantitativo, multivariado descriptivocorrelacional, catalogado de naturaleza descriptiva del fenómeno y las percepciones generales de la muestra según el constructo estudiado.

La instrumentación se realizó por medio de la adaptación a la realidad chilena del "Cuestionario para futuros docentes de Educación Secundaria acerca de las percepciones sobre atención a la diversidad” (Colmenero y Pegalajar, 2015), del que se tomaron y adaptaron tres variables (elementos condicionantes del proceso de inclusión educativa, 
evaluación de la formación docente en relación a la inclusión educativa y práctica docente formativa hacia la inclusión educativa) de las cinco consideradas en el cuestionario original (elementos condicionantes del proceso de atención a la diversidad en el aula, respuesta curricular y organizativa en el aula, capacitación docente hacia la diversidad, práctica docente formativa en la atención a la diversidad y percepción docente hacia el alumnado con necesidades específicas de apoyo educativo), ya que las dos no contempladas, corresponden a factores propios de la formación docente de un escenario específico de la educación superior española.

El cuestionario fue adaptado y validado para este estudio por medio de Análisis Factorial. Los resultados en donde la validez de constructo se expresa en las pruebas de KMO $=0,80$ con p < 0,001 apegándose y justificándose el tipo de análisis (Nunnally, 1973, 1987; Kerlinger y Lee, 2002; Caro, Escalante y Barahona, 2002), para el cuestionario original, toman los valores de $\mathrm{KMO}=0,68$ con $\mathrm{p}<0,001$ que refleja un valor que justifica el tipo de cálculos realizados (Caro, Escalante y Barahona, 2002; Kerlinger y Lee, 2002; Nunnally, 1973, 1987), aun existiendo diferencias con el valor del cuestionario original. También se mostró que la rotación de los componentes principales por medio de rotación Varimax del $60,8 \%$ del total de la varianza explicada para los cinco factores incluidos en el cuestionario original, obteniéndose los por medio de rotación de los componentes principales por medio de rotación Varimax un valor de 51,9\% del total de la varianza total explicada para los tres factores contemplados en el cuestionario adaptado.

Se desarrollaron pruebas complementarias como indicadores del ajuste del cuestionario, donde se resultados para el cálculo de Chi-cuadrado de las tres variables seleccionadas y ajustadas, para la variable Elementos condicionantes del proceso de inclusión educativa Chi-cuadrado $=410,95$ con $\mathrm{p}<0,01$ y 360 grados de libertad, para la variable Formación docente en relación a la inclusión educativa Chi-cuadrado $=567,60$ con $\mathrm{p}<0,01$ y 420 grados de libertad, para la variable Práctica docente formativa hacia la inclusión Chicuadrado $=863,52$ con $\mathrm{p}<0,01$ y 570 grados de libertad. Los resultados obtenidos en las pruebas de Chi-cuadrado, permiten validar y aceptar la adaptación la variable Elementos condicionantes del proceso de inclusión educativa, no así las variables Formación docente en relación a la inclusión educativa, las que poseen valores superiores a los planteados para los respectivos grados de libertad y $p$, aun siendo estos valores cercanos a los ideales.

La confiabilidad expresada para el cuestionario original, por medio del Alfa de Cronbach es de 0,92, lo que según la teoría psicométrica se considera como Excelente, los cálculos de confiabilidad para el cuestionario adaptado, obtuvieron el valor de 0,756 para Alfa de Cronbach, el que se considera como Alto según la teoría psicométrica (Caro, Escalante y Barahona, 2002; Kerlinger y Lee, 2002; Nunnally, 1973, 1987).

De la misma manera se midió la confiabilidad para el total de ítems para cada una de las variables consideradas, los hallazgos encontrados se apegan a lo descrito como confiabilidad total del instrumento en donde los resultados fueron los siguientes: Elementos condicionantes del proceso de inclusión educativa, Alfa de Cronbach 0,74 para los 17 ítems planteados. Formación docente en relación a la inclusión educativa, Alfa de Cronbach 0,789 para los cinco ítems planteados. Práctica docente formativa hacia la inclusión educativa, Alfa de Cronbach 0,80 para los siete ítems planteados.

Las variables adaptadas fueron: (V1) Elementos condicionantes del proceso de inclusión educativa; que describe los elementos presentes en el proceso de enseñanza y aprendizaje que el docente debe considerar para desarrollar un proceso de atención a la diversidad de 
calidad en el aula, (V2) Formación docente en relación a la inclusión educativa; que se centra en analizar el nivel formativo recibido por el docente para dar respuesta eficaz a las necesidades educativas y (V3) Práctica docente formativa hacia la inclusión educativa; que analiza el modo en que la formación recibida permite al futuro docente responder a los intereses e inquietudes detectados en el alumnado con necesidades específicas de apoyo educativo (cuadro 1).

Cuadro 1. Especificaciones del cuestionario adaptado

\begin{tabular}{clc}
\hline ÍTEMS & \multicolumn{1}{c}{ VARIABLE } & N DE ÍTEMS $^{\circ}$ \\
\hline \multirow{2}{*}{ al 17} & $\begin{array}{l}\text { V1. Elementos condicionantes del proceso de inclusión } \\
\text { educativa }\end{array}$ & 17 \\
18 al 22 & $\begin{array}{l}\text { V2. Evaluación de la formación docente en relación a la } \\
\text { inclusión educativa }\end{array}$ & 5 \\
23 al 29 & V3. Práctica docente formativa hacia la inclusión educativa & 8 \\
\hline
\end{tabular}

Fuente: Elaboración propia.

Los ítems se plantearon en forma de afirmaciones teniendo como posibles respuestas la manifestación de estar Muy de acuerdo, De acuerdo, En desacuerdo y Muy en desacuerdo (cuadro 2).

Cuadro 2. Ejemplos de reactivos

\begin{tabular}{|c|c|c|c|c|}
\hline & 1 & 2 & 3 & 4 \\
\hline 1. Es un deber de las escuelas atender a todos los estudiantes. & & & & \\
\hline $\begin{array}{l}\text { 3. La atención a la inclusión educativa en el aula enriquece a toda la comunidad } \\
\text { educativa. }\end{array}$ & & & & \\
\hline $\begin{array}{l}\text { 16. Un proceso de inclusión educativa de calidad requiere de motivación o } \\
\text { interés profesional docente. }\end{array}$ & & & & \\
\hline $\begin{array}{l}\text { 20. Las actividades o ejemplos prácticos desarrollados en las clases han mejorado } \\
\text { mis conocimientos sobre la inclusión educativa. }\end{array}$ & & & & \\
\hline $\begin{array}{l}\text { 26. Trabajar con estudiantes con necesidades educativas especiales es un trabajo } \\
\text { extra para los profesores. }\end{array}$ & & & & \\
\hline $\begin{array}{l}\text { 28. Un estudiante con necesidades educativas especiales, interrumpe la rutina } \\
\text { del aula y perjudica el aprendizaje de sus compañeros. }\end{array}$ & & & & \\
\hline
\end{tabular}

Nota: 1: Muy de acuerdo. 2: De acuerdo. 3: En desacuerdo. 4: Muy en desacuerdo.

Fuente: Elaboración propia.

El estudio se realizó de manera censal para una universidad pública con 152 estudiantes y el cuestionario se aplicó a una muestra de121 estudiantes que representan al 76,61\% del universo compuesto por los estudiantes de las carreras de Pedagogía en Matemáticas, Pedagogía en inglés, Educación Parvularia con mención en inglés, Pedagogía General Básica con mención en inglés, Pedagogía General Básica con mención en alemán y Educación Parvularia con mención en alemán. Las diferencias en los $\mathrm{N}$ indicados, tienen relación directa con la aplicación del cuestionario en la calendarización de esta, ya que la posibilidad de ausentismo de los estudiantes, no puede ser determinada por quienes aplican los cuestionarios, de igual manera se considera que la muestra final obtenida es significativa por el porcentaje del universo que representa.

Para el correcto logro de la aplicación del cuestionario se realizó el proceso de consentimiento informado, por grupo de aplicación, el que fue realizado por uno de los miembros del equipo de investigación de manera oral, en donde se indicó el título de la investigación, los nombres del equipo de trabajo, los objetivos de la investigación y se realizó el compromiso ético de protección de los datos y mantención del anonimato de cada una de las personas que respondieron el cuestionario. 


\section{Resultados}

El peso de las variables consideradas se calculó por medio de correlación de Pearson observándose qué para el constructo planteado, las influencias de las diferentes variables consideradas (cuadro 3). La variable que mayor peso posee en las percepciones de los estudiantes de pedagogía sobre la inclusión educativa es la Práctica docente formativa hacia la inclusión educativa, con un valor de correlación de 0,78. Seguido de la Formación docente en relación a la inclusión educativa, con un valor de 0,67. Y los Elementos condicionantes del proceso de inclusión educativa, con un valor de 0,42. Los valores calculados fueron obtenidos por medio del cálculo de correlaciones de Pearson, significativa a nivel de 0,01 bilateral para valores absolutos.

Los cálculos de los puntajes obtenidos se desarrollaron sobre un valor total ideal de 116 puntos, y puntajes ideales para cada una de las variables; Elementos condicionantes del proceso de inclusión educativa (68 puntos). Formación docente en relación a la inclusión educativa (20 puntos). Y Práctica docente formativa hacia la inclusión educativa (28 puntos). Obteniendo valores considerados altos en la totalidad de las variables, pero con diferencias notorias entre las diferentes carreras seguidas por los estudiantes que contestaron el cuestionario (cuadro 4).

Cuadro 3. Correlaciones intervariables

\begin{tabular}{lllccc}
\hline & & V1 & V2 & V3 & TOTAL \\
\hline $\begin{array}{l}\text { V1. Elementos condicionantes del proceso } \\
\text { de inclusión educativa }\end{array}$ & Corr.de Pearson & 1 & 0,006 & 0,002 & $0,420^{*}$ \\
& Sig. (bilateral) & & 0,950 & 0,980 & 0,000 \\
\hline V2. Formación docente en relación a la & Corr.de Pearson & 0,006 & 1 & $0,280^{*}$ & $0,670^{*}$ \\
inclusión educativa & Sig. (bilateral) & 0,95 & 0,002 & 0,000 \\
\hline V3. Práctica docente formativa hacia la & Corr.de Pearson & 0,002 & $0,280^{*}$ & 1 & $0,780^{*}$ \\
inclusión educativa & Sig. (bilateral) & 0,98 & 0,002 & & 0,000 \\
\hline \multirow{2}{*}{ Total } & Corr.de Pearson & $0,420^{*} 0,670^{*} 0,780^{*}$ & 1 \\
& Sig. (bilateral) & 0,000 & 0,000 & 0,000 & \\
\hline
\end{tabular}

Nota: ${ }^{*}$ La correlación es significativa $\mathrm{p}<0,01$ (bilateral). $\mathrm{N}=121$.

Fuente: Elaboración propia.

Los valores alcanzados por los estudiantes de las distintas carreras, se expresan para las percepciones de los estudiantes sobre la inclusión educativa, expresado en el análisis como Total, posee un orden en donde el puntaje mayor alcanzado es de 103 puntos para Educación Parvularia con mención en inglés con $\mathrm{N}=11$, en segundo lugar se encuentra Pedagogía en Educación Básica con mención en inglés con 99,7 puntos y $\mathrm{N}=11$, Pedagogía en Matemáticas posee el puntaje posicionado en tercer lugar con 99,7 puntos y $\mathrm{N}=20$, el cuarto lugar de los puntajes es de Educación Básica con mención en alemán con 97,4 puntos con $\mathrm{N}=3$, Pedagogía en inglés con 96,9 puntos y $\mathrm{N}=65$, y con 96,7 puntos y $\mathrm{N}=3$ Educación Parvularia con mención en alemán posee el menor puntaje alcanzado en la aplicación del instrumento. Para complementar lo descrito anteriormente es que se desarrolló la prueba ANOVA de un factor obteniendo los siguientes resultados (cuadro 5).

Los resultados para el cálculo de $\mathrm{F}$ y su interpretación, muestra que las diferencias de medias se clasifican de más significativas a menos significativas, teniendo en primer lugar a las pertenecientes a la XV1 donde $\mathrm{F}=2,37$, seguida de $\mathrm{XV} 2$ donde $\mathrm{F}=2,1$ y XV 3 donde $\mathrm{F}=1,38$, para los puntajes totales, encontramos que las diferencias poseen un valor $\mathrm{F}=$ 2,14 . 
Cuadro 4. Resultados según carrera

\begin{tabular}{llrrrr}
\hline & & V1 & V2 & V3 & TOTAL \\
\hline \multirow{2}{*}{ Pedagogía en matemáticas } & Media & 65,4 & 14,10 & 20,20 & 99,70 \\
& $\mathrm{~N}$ & 20 & 20 & 20 & 20 \\
& Dt. & 3,36 & 2,94 & 4,32 & 6,42 \\
\hline \multirow{2}{*}{ Pedagogía en inglés } & Media & 65,5 & 13,50 & 17,80 & 96,90 \\
& $\mathrm{~N}$ & 65 & 65 & 65 & 65 \\
& Dt. & 2,68 & 3,55 & 4,12 & 6,86 \\
\hline \multirow{2}{*}{ Educación Parvularia } & Media & 67,70 & 16,30 & 19,20 & 103 \\
mención en inglés & $\mathrm{N}$ & 11 & 11 & 11 & 11 \\
& Dt. & 0,47 & 2,69 & 5,02 & 6,62 \\
\hline \multirow{2}{*}{ Pedagogía General Básica } & Media & 66,50 & 15,10 & 18,20 & 99,70 \\
mención en inglés & $\mathrm{N}$ & 11 & 11 & 11 & 11 \\
& Dt. & 1,29 & 3,83 & 4,66 & 6,74 \\
\hline \multirow{2}{*}{ Pedagogía General Básica } & Media & 64 & 15,70 & 17,70 & 97,40 \\
& $\mathrm{~N}$ & 11 & 11 & 11 & 11 \\
& Dt. & 3,89 & 1,90 & 4,43 & 5,77 \\
\hline \multirow{2}{*}{ Educación en Parvularia } & Media & 66,30 & 15 & 15,30 & 96,70 \\
mención en alemán & $\mathrm{N}$ & 3 & 3 & 3 & 3 \\
& Dt. & 2,08 & 0,00 & 0,58 & 1,53 \\
\hline \multirow{2}{*}{ Total } & Media & 65,7 & 14,30 & 18,30 & 98,20 \\
& $\mathrm{~N}$ & 121 & 121 & 121 & 121 \\
& Dt. & 2,80 & 3,34 & 4,31 & 6,77 \\
\hline
\end{tabular}

Fuente: Elaboración propia.

Cuadro 5. ANOVA de un factor

\begin{tabular}{|c|c|c|c|c|c|c|}
\hline & & $\begin{array}{c}\text { SUMA DE } \\
\text { CUADRADOS }\end{array}$ & GL & $\begin{array}{c}\text { MEDIA } \\
\text { CUADRÁTICA }\end{array}$ & $\mathbf{F}$ & SIG. \\
\hline \multirow{3}{*}{$\mathrm{XV} 1$} & Inter-grupos & 87,90 & 5 & 17,60 & 2,37 & 0,040 \\
\hline & Intra-grupos & 852,50 & 115 & 7,41 & & \\
\hline & Total & 940,40 & 120 & & & \\
\hline \multirow{3}{*}{$\mathrm{XV} 2$} & Inter-grupos & 111,80 & 5 & 22,40 & 2,10 & 0,070 \\
\hline & Intra-grupos & 1227,20 & 115 & 10,70 & & \\
\hline & Total & 1339,10 & 120 & & & \\
\hline \multirow{3}{*}{ XV3 } & Inter-grupos & 126,20 & 5 & 25,20 & 1,38 & 0,240 \\
\hline & Intra-grupos & 2105,10 & 115 & 18,30 & & \\
\hline & Total & 2231,30 & 120 & & & \\
\hline \multirow{3}{*}{ Total } & Inter-grupos & 467,90 & 5 & 93,60 & 2,14 & 0,070 \\
\hline & Intra-grupos & 5027,70 & 115 & 43,720 & & \\
\hline & Total & 5495,50 & 120 & & & \\
\hline
\end{tabular}

Fuente. Elaboración propia.

De la misma forma al fijarnos en los puntajes alcanzados para las variables consideradas, y la relación de estos con la promoción, teniendo como años de ingreso el $2014(\mathrm{~N}=1)$, $2015(\mathrm{~N}=26), 2016(\mathrm{~N}=40)$ y $2017(\mathrm{~N}=54)$, con un total de $\mathrm{N}=121$ para los cuestionarios respondidos (cuadro 6).

$\mathrm{Al}$ revisar los puntajes alcanzados para las diferentes variables y totales, se observa que para los Elementos condicionantes del proceso de inclusión educativa, el puntaje mayor $68(\mathrm{~N}=1)$ para la promoción 2014, representa al $0,82 \%$ de la muestra, no siendo significativo, los siguientes puntajes en orden de mayor a menos son: 66,8 $(\mathrm{N}=54)$ representando al 44,6\% de la muestra la promoción 2017 muestra un mayor reconocimiento de la variable $65,1(\mathrm{~N}=40)$ en la promoción 2016 , es un $33,1 \%$ de la muestra y $64,1(\mathrm{~N}=26)$, promoción 2015 representa un $21,5 \%$ de la muestra. 
Cuadro 6. Resultados según promoción

\begin{tabular}{|c|c|c|c|c|c|}
\hline & & $\begin{array}{l}\text { ELEMENTOS COND. } \\
\text { DEL PROCESO DE } \\
\text { INCLUSIÓN ED. }\end{array}$ & $\begin{array}{l}\text { FORMACIÓN } \\
\text { DOCENTE EN } \\
\text { INCLUSIÓN ED. }\end{array}$ & $\begin{array}{c}\text { PRÁCTICA } \\
\text { DOC.FORMAT. LA } \\
\text { INCLUSIÓN ED. }\end{array}$ & TOTAL \\
\hline \multirow{3}{*}{2014} & Media & 68,00 & 15,00 & 15 & 98 \\
\hline & $\mathrm{N}$ & 1 & 1 & 1 & 1 \\
\hline & Dt. & . & . & . & . \\
\hline \multirow{3}{*}{2015} & Media & 64,10 & 14,70 & 18,90 & 97,70 \\
\hline & $\mathrm{N}$ & 26 & 26 & 26 & 26 \\
\hline & Dt. & 3,68 & 3,61 & 4,71 & 8,96 \\
\hline \multirow{3}{*}{2016} & Media & 65,10 & 14,10 & 18,80 & 98,10 \\
\hline & $\mathrm{N}$ & 40 & 40 & 40 & 40 \\
\hline & Dt. & 2,86 & 3,09 & 4,10 & 5,81 \\
\hline \multirow{3}{*}{2017} & Media & 66,80 & 14,10 & 17,70 & 98,60 \\
\hline & $\mathrm{N}$ & 54 & 54 & 54 & 54 \\
\hline & Dt. & 1,60 & 3,46 & 4,27 & 6,39 \\
\hline \multirow{3}{*}{ Total } & Media & 65,70 & 14,30 & 18,30 & 98,20 \\
\hline & $\mathrm{N}$ & 121 & 121 & 121 & 121 \\
\hline & Dt. & 2,80 & 3,34 & 4,31 & 6,77 \\
\hline
\end{tabular}

Fuente: Elaboración propia.

La Formación docente en relación a la inclusión educativa, sigue mostrando como mayor puntaje a la generación 2014 $(\mathrm{N}=1)$, no representativo estadísticamente, seguido de la promoción $2015(\mathrm{~N}=26)$ con 14,7 puntos, la promoción $2016(\mathrm{~N}=40)$ con 14,1 puntos y la promoción $2017(\mathrm{~N}=54)$ con 14,1 puntos.

El desarrollo de una Práctica docente formativa hacia la inclusión educativa, muestra con un mayor puntaje a la promoción $2015(\mathrm{~N}=26)$ con puntaje 18,9. En segundo lugar, a la promoción $2016(\mathrm{~N}=40)$ con 18,8 puntos y la promoción $2017(\mathrm{~N}=54)$ que obtuvo como puntaje 17,7 , la promoción $2014(\mathrm{~N}=1)$, aun siendo no significativa a nivel estadístico, muestra un puntaje de 15 , el más bajo en esta variable.

Para los puntajes totales alcanzados, se excluye a la promoción 2014, quedando en un orden de mayor a menor, las promociones 2017, 2016 y 2015, con puntajes 98,6, 98,1 y 97,7 respectivamente, la promoción excluida en esta etapa, obtuvo un puntaje de 98 . Para complementar lo descrito anteriormente es que se desarrolló la prueba ANOVA de un factor donde se visualizaron los siguientes resultados (cuadro 7).

Cuadro 7. ANOVA de un factor

\begin{tabular}{|c|c|c|c|c|c|c|}
\hline & & $\begin{array}{c}\text { SUMA DE } \\
\text { CUADRADOS }\end{array}$ & GL & $\begin{array}{c}\text { MEDIA } \\
\text { CUADRÁTICA } \\
\end{array}$ & $\mathbf{F}$ & SIG. \\
\hline \multirow{3}{*}{ XV1 } & Inter-grupos & 148,60 & 3 & 49,50 & 7,32 & 0,000 \\
\hline & Intra-grupos & 791,80 & 117 & 6,77 & & \\
\hline & Total & 940,40 & 120 & & & \\
\hline \multirow{3}{*}{$\mathrm{XV} 2$} & Inter-grupos & 8,23 & 3 & 2,74 & 0,24 & 0,870 \\
\hline & Intra-grupos & 1330,80 & 117 & 11,38 & & \\
\hline & Total & 1339,10 & 120 & & & \\
\hline \multirow{3}{*}{ XV3 } & Inter-grupos & 53,50 & 3 & 17,80 & 0,96 & 0,420 \\
\hline & Intra-grupos & 2177,80 & 117 & 18,60 & & \\
\hline & Total & 2231,30 & 120 & & & \\
\hline \multirow{3}{*}{ Total } & Inter-grupos & 13,60 & 3 & 4,53 & 0,09 & 0,960 \\
\hline & Intra-grupos & 5481,90 & 117 & 46,80 & & \\
\hline & Total & 5495,50 & 120 & & & \\
\hline
\end{tabular}

Fuente: Elaboración propia. 
Los resultados para la prueba $\mathrm{F}$, muestran que la variable que posee diferencias más significativas es XV1, donde $\mathrm{F}=7,32$, también se muestra que las variables XV2 y XV3, no poseen diferencias mayormente significativas, ya que en ambas el valor de $\mathrm{F}$ es inferior a 1, siendo $\mathrm{F}=0,24$ para $\mathrm{XV} 2$ y $\mathrm{F}=0,42$ para XV3, en los puntajes totales alcanzados, se considera que las diferencias de media existentes no son significativas ya que el valor de F para total es $\mathrm{F}=0,097$.

\section{Conclusión}

Podemos afirmar que teniendo un alcance positivo $(84,7 \%)$ en la percepción hacia la inclusión educativa, esta mostró diferencias significativas en las diferentes variables que la componen, donde la percepción más positiva es sobre los elementos que condicionan y definen la inclusión educativa (96,6\%), seguido de la percepción de la formación docente en relación a la inclusión educativa (71,3\%), quedando en último lugar la percepción de las practicas docentes formativas hacia la inclusión educativa, en donde los estudiantes muestran un alcance más bajo (65,5\%).

La consideración de un constructo compuesto por variables de diferente naturaleza, mostró que cada una de las variables posee un peso determinado con el cual se confirma su pertenencia y consideración en la medición realizada, siendo la variable de mayor peso la práctica docente formativa hacia la inclusión educativa que representa un $77,6 \%$ del concepto global, seguida de la formación docente en relación a la inclusión educativa que representa un $67,4 \%$ y los elementos condicionantes del proceso de inclusión educativa, que solo representan el $41,8 \%$ del concepto. Lo que mostraría una inclinación de los estudiantes universitarios por el desarrollo de prácticas que fortalezcan la inclusión, ya no desde la teoría, más bien pensando en el rol de interacción constante que desempeñarán en su rol de educadores.

La caracterización de las diferentes carreras reflejó que las percepciones positivas en relación a la inclusión educativa mantienen un orden, en donde los primeros lugares los ocupan las carreras que no corresponden a especialidades, por lo que las ciencias especificas se ubican en un segundo lugar y los estudiantes de carreras pertenecientes a pedagogías en idiomas o con mención en idiomas que no sea inglés, poseen el menor puntaje. Sobre el año de ingreso de los estudiantes, para las percepciones de los estudiantes sobre la inclusión educativa, se consideran tres años de ingreso, donde mientras más cercano el año de ingreso, los estudiantes poseen percepciones más positivas hacia la inclusión educativa, lo que coincide con la considerada variable 1 (elementos que condicionan y definen la inclusión educativa) y que discrepan totalmente con las variables 2 y 3 (Formación docente en relación a la inclusión educativa y Práctica docente formativa hacia la inclusión educativa), las que ordenan de manera inversa sus resultados.

También podemos afirmar que, existiendo diferencias en las medias alcanzadas por las diferentes carreras impartidas y los diferentes años de ingreso, éstas son mayormente significativas a nivel de comparación en las diferentes carreras, donde la variable Elementos condicionantes del proceso de inclusión educativa, posee un mayor valor para F, seguido de las variables Formación docente en relación a la inclusión educativa y Práctica docente formativa hacia la inclusión educativa respectivamente. Sobre los resultados obtenidos en la búsqueda de diferencias significativas a nivel de los años de ingreso de los estudiantes, los resultados muestran que el valor $\mathrm{F}$ es significativo solo para 
los Elementos condicionantes del proceso de inclusión educativa, siendo el único valor obtenido mayor que 1, donde las variables Formación docente en relación a la inclusión educativa y Práctica docente formativa hacia la inclusión educativa y el valor otorgado al cuestionario completo, no se consideran significativos.

Finalmente, y a modo de reflexión, los presentes resultados son una aproximación al análisis de las complejas relaciones que se establecen en la formación de futuros profesores, sus actitudes hacia la inclusión educativa y sus posibles actuaciones en un contexto de práctica pedagógica, por lo que deben tomarse con cautela al poseer la limitación de una muestra contextualizada a una facultad de reciente creación. Sin embargo, entrega elementos que se pueden llevar a discusión por todos los actores que quieren un cambio de paradigma hacia una sociedad que se reconstruya mirando de frente la diversidad, interculturalidad e inclusión de todos y todas.

Como plantea la UNESCO (2015), se hace necesario replantear-nos los fines de la educación y la construcción del conocimiento en un mundo cambiante y complejo, y para ello la figura de los educadores seguirán siendo factor de cambio y transformación. Las posibilidades de un desarrollo sostenible e inclusivo nos deben mover a centrarnos en nuevos enfoques de aprendizaje para todos y todas, y que propicien la equidad social y solidaridad mundial.

La labor de atención a la inclusión urge y se hace necesario que las instituciones formadoras de profesores consideren las variables descritas para la mejora de sus recorridos profesionales, tanto a nivel curricular como extra-curricular. Las Instituciones de Educación Superior a través de la Formación Inicial Docente requiere de una práctica constante en temas de inclusión y atención a la diversidad y para ello debe tender puentes con la Formación Permanente de Profesores, los gremios de docentes, los administradores de la educación, organizaciones de base y por sobre todo las escuelas, de manera tal de asegurar una continuidad de las innovaciones y procesos de mejora que se persiguen para una nueva educación pública de calidad y con real sentido de justicia social.

\section{Referencias}

Ainscow, M., Booth, T. y Dyson, A. (2006). Improving schools, developing inclusion. Londres: Routledge.

Caro, A., Escalante, E. y Barahona, L. (2002). Análisis y tratamiento de datos en SPSS. Valparaíso: Ediciones Universidad de Playa Ancha.

Colmenero, M. J. y Pegalajar, M. C. (2015). Cuestionario para futuros docentes de educación secundaria acerca de las percepciones sobre atención a la diversidad: Construcción y validación del instrumento. Estudios sobre Educación, 29, 165-189. https://doi.org/10.15581/004.29.165-189

Chiner, E. (2011). Las percepciones y actitudes del profesorado hacia la inclusión del alumnado con necesidades educativas especiales como indicadores del uso de prácticas educativas inclusivas en el aula. Tesis Doctoral. Universidad de Alicante, España.

Echeita, G. y Sandoval, M. (2002). Educación inclusiva o educación sin exclusiones. Revista de Educación, 327, 31-48.

Escribano, A. y Martínez, A. (2013). Inclusión educativa y profesorado inclusivo: aprender juntos para aprender a vivir juntos. Madrid: Narcea. 
Essomba, M. (2006). Liderar escuelas interculturales e inclusivas: Equipos directivos y profesorado ante la diversidad cultural y la inmigración. Barcelona: GRAÓ.

Esteve, J. (2003). La tercera revolución educativa: La educación en la sociedad del conocimiento. Barcelona: Paidós Ibérica.

González-Gil, F., Martin-Pastor, E., Poy, R. y Jenaro, C. (2016). Percepciones del profesorado sobre la inclusión: estudio preliminar. Profesorado. Revista Electrónica Interuniversitaria de Formación del Profesorado, 19(3), 11-24. http://dx.doi.org/10.6018/reifop.19.3.219321

Infante, M. (2010). Desafíos a la formación docente: Inclusión educativa. Estudios Pedagógicos, 36(1), 287-297. https://dx.doi.org/10.4067/S0718-07052010000100016

Kerlinger, E. y Lee, H. (2002). Investigación del comportamiento. Ciudad de México: McGraw-Hill.

López, V., Julio, C., Pérez, M.V., Morales, M. y Rojas, C. (2014). Barreras culturales para la inclusión: Políticas y prácticas de integración en Chile. Revista de Educación, 363, 256-281. http://dx.doi.org/10.4438/1988-592X-RE-2012-363-180

Manghi, D., Julio, C., Conejeros, M., Donoso, E., Murillo, M. y Díaz, C. (2012). El profesor de educación diferencial en Chile para el siglo XXI: Tránsito de paradigma en la formación profesional. Revista Perspectiva Educacional, 51(2), 46-71. Recuperado de http://www.redalyc.org/articulo.oa?id=333328168004

Mateus, L., Vallejo, D., Obando, D. y Fonseca, L. (2017). Percepción de las prácticas y de la cultura inclusiva en una comunidad escolar. Avances en Psicología Latinoamericana, 35(1), 177-191. https://dx.doi.org/10.12804/revistas.urosario.edu.co/apl/a.4854.

MINEDUC. (2007). Calidad de la integración escolar y factores asociados. Recuperado de http://especial.mineduc.cl/wp-content/

MINEDUC. (2008). Marco para la buena enseñanza. Recuperado de http://www.cpeip.cl/wpcontent/uploads/2017/10/MBE.pdf

MINEDUC. (2015). Ley $N^{\circ}$ 20.845. Ley de inclusión escolar que regula la admisión de los y las estudiantes, elimina el financiamiento compartido y prohíbe el lucro en establecimientos educacionales que reciben aportes del estado. Recuperado de https://www.leychile.cl/Navegar?idNorma=1078172

Nunnally J. (1973). Introducción a la medición psicológica. Ciudad de México: Centro Regional de Ayuda Técnica.

Nunnally J. (1987). Teoría psicométrica. Ciudad de Mexico: Trillas.

OCDE. (2009). Informe TALIS. La creación de entornos eficaces de enseñanza y aprendizaje. Síntesis de los primeros resultados. Recuperado de http://www.ub.edu/obipd/docs/educacionp/Informes/33.pdf

ONU/CEPAL. (2016). Agenda 2030 y los Objetivos de Desarrollo Sostenible. Una oportunidad para América Latina y el Caribe. Santiago de Chile: CEPAL.

Richardson, V. (1996). The role of attitudes and beliefs in learning to teach. En J. Sikula (Ed.), Handbook of research on teacher education (pp. 102-119). Nueva York, NY: Macmillan.

Sanhueza, S., Granada, M. y Bravo, L. (2012). Actitudes del profesorado de Chile y Costa Rica hacia la inclusión educativa. Cadernos de Pesquisa, 42(147), 884-899. https://doi.org/10.1590/SO100-15742012000300013

Slee, R. (2001). Inclusion in practice: Does practice make perfect? Educational Review, 53, 113-123. https://doi.org/10.1080/00131910120055543 
Slee, R. y Allan, J. (2001). Excluding the included: A reconsideration of inclusive education. International Studies in Sociology of Education, 11(2), 173-192, https://doi.org/10.1080/09620210100200073

Stainback, S. y Stainback, W. (1999). Aulas inclusivas: Un nuevo modo de enfocar y vivir el currículo. Madrid: Narcea.

Tenorio, S. (2011). Formación inicial docente y necesidades educativas especiales. Estudios Pedagógicos, 37(2), 249-265. https://doi.org/10.4067/S0718-07052011000200015

UNESCO. (1990). Declaración mundial sobre educación para todos y el marco de acción para satisfacer las necesidades básicas de aprendizaje. París: UNESCO.

UNESCO. (1994). Declaración sobre necesidades educativas especiales: Acceso y calidad de Salamanca. París: UNESCO.

UNESCO. (2000). Foro mundial educación para todos. París: UNESCO.

UNESCO. (2005). Guidelines for inclusion: Ensuring access to education for all. París: UNESCO.

UNESCO. (2009). Conferencia internacional de educación 48 reunión: La educación inclusiva: Un camino hacia el futuro. París: UNESCO.

UNESCO. (2015). Replantear la educación. ¿Hacia un bien común mundial? París: UNESCO.

\section{Breve CV de los autores}

\section{Pablo Castillo Armijo}

Doctor en Educación y Sociedad, Máster Oficial en Investigación en Didáctica, Formación y Evaluación Educativa y Licenciado en Pedagogía por la Universitat de Barcelona, España. Título de Profesor de Historia y Geografía, Licenciado en Historia y Magíster en Educación con Mención en Currículum por la Pontificia Universidad Católica de Valparaíso, Chile. Profesor Conferenciante de la Facultad de Ciencias de la Educación e Investigador Postdoctoral del Núcleo Científico Multidisciplinario de la Dirección de Investigación de la Universidad de Talca-Chile. ORCID ID: https://orcid.org/oooo0002-5708-4618. Email: pacastillo@utalca.cl

\section{Carlos Miranda Carvajal}

Magíster en Evaluación Educacional, Título de Profesor de Educación General Básica con Mención en Educación y Desarrollo Rural y Licenciado en Educación por la Universidad de Playa Ancha, Chile. Diplomado en Educación Intercultural Bilingüe, Cátedra Indígena, Universidad de Chile. Actualmente se desarrolla como profesor de la escuela Teniente Julio Allende Ovalle de Valparaíso, como consultor en Genium Consultores e Investigador colaborador de la Facultad de Ciencias de la Educación de la Universidad de Talca. ORCID ID: https://orcid.org/0000-0003-0570-3496. Email: cmc.mira@gmail.com 